WEBLEY, D. M. (1953). J. gen. Microbiol. 8, 66-71.

\title{
A Simple Method for Producing Microcultures in Hanging Drops with special reference to Organisms Utilizing Oils
}

\author{
By D. M. WEBLEY \\ WITH A NOTE BY V. C. FARMER \\ Macaulay Institute for Soil Research, Craigiebuckler, Aberdeen
}

SUMMARY: An aseptic technique is described for obtaining small hanging drops on the surface of coverslips, using only apparatus that is cheap and easy to obtain. The technique has been used to demonstrate the growth of Nocardia salmonicolor on liquid paraffin droplets.

Of all the methods used for studying the growth and development of microorganisms in liquid culture in undisturbed condition the hanging drop is probably the best. The ordinary hanging-drop method, as used for routine examination of gross bacterial morphology and motility, yields unavoidably large drops and is unsuited to the study of the growth and development of a few cells. Microbiologists requiring more stringent control of drop size have turned to the technique of micromanipulation, but for the average worker the apparatus required is too time-consuming and tedious to construct. The technique described below is offered for those workers who do not require the refinements of micromanipulation methods. The apparatus required is cheap and easy to obtain.

\section{ME'THOD}

The method consists of spraying a suspension of organisms, in the medium in which they are to be studied, on to the surface of coverslips. The suspension is made of such a density that the droplets formed on the coverslip surface contain a few cells each. The coverslip is then inverted over a well slide containing liquid paraffin and incubated at the required temperature.

\section{Description of apparatus and technique}

The spray. An all-glass spray of the type depicted in Fig. 1 (manufactured by Parke, Davis and Co., Hounslow, Middlesex, under the trade name Glaseptic Nebuliser) is used. It is plugged as shown and sterilized empty, Fig. 1, A. The suspension of organisms is introduced aseptically at the top of the spray. The squeeze bulb is then attached. Another type of spray manufactured by Parke, Davis and Co. has a slightly different design (Fig. 1, B). If this is used it is an advantage to attach a short piece of glass tubing carrying a cotton-wool plug (see Fig. 1, B) to the side arm by a short piece of rubber tubing before sterilization. In all other essentials the spray is the same as the type depicted in Fig. 1, A.

Moist chamber. Owing to rapid evaporation of microdrops, it is necessary 
to spray in an enclosed humid atmosphere. Fig. 2 illustrates a type of moist chamber which has given good results. It is made from: (1) A porcelain lampshade $(A)$ with a small piece removed to allow free passage of the rubber tubing attaching the squeeze bulb to the side of the spray; (2) a cylindrical glass jar open at both ends $(B) ;(3)$ a circular glass plate $(C)$ with a hole of about $\frac{1}{4}$ in. in diameter drilled in the centre; and (4) a glass ring $(D)\left(\frac{5}{8}\right.$ in. in diameter $\times \frac{1}{4}$ in. in height). The parts are assembled as illustrated in Fig. 2.

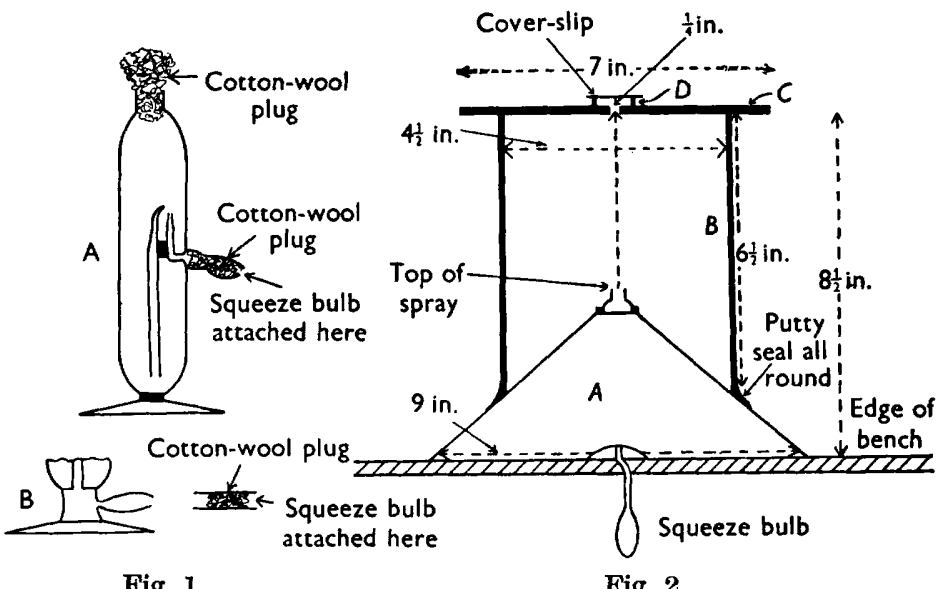

Fig. 1

Fig. 2

$B$ is sealed by a ring of putty to the side of the lampshade and is lined with moist filter-paper. $C$ can be sealed with liquid paraffin at its contact with the top edge of $B$; the circular hole in $C$ is aligned with the top of the spray when the latter is in position. The glass ring $D$ is placed so that the circular hole in $C$ is in the centre of the area covered by $D$. $D$ is kept in position by liquid paraffin between the surface of $C$ and its edge. It is advisable to use a fresh sterile circular ring, $D$, for each experiment. These can be sterilized in Petri dishes. When required, a ring can be sealed in position over the orifice in $C$ just before the coverslip is placed in position (see Fig. 2), the surface of $C$ being swabbed just prior to the placing of $D$ and its coverslip.

Preparation of coverslips. To obtain well-formed droplets with a circular edge it is necessary to coat the coverslips with a water-repellent film. For this purpose, Reyniers (1933) and Gee \& Hunt (1928) prepared coverslips with a thin grease film. While this method can be employed quite satisfactorily, it has been found that more consistent results requiring very little experience can be obtained by coating the coverslips with a silicone water-repellent film. For a discussion of the use of silicone water repellents the reader is referred to Gilbert (1951). The method used in this investigation is as follows: no. 1 coverslips $\frac{7}{8}$ in. square are thoroughly cleaned in hot chromic-sulphuric acid cleaning mixture. After removal from the cleaning solution they are well washed by several passages first under running water and finally through distilled water after which they are stored in $50 \%$ ethanol. Before application 
of the silicone film, the coverslips are removed from the ethanol and dried with acetone. When completely dry they are treated with a silicone impregnated tissue; 'lens cleaners' (Macleans' Ltd., Brentford, Middlesex) are very satisfactory. The coverslip is placed between the folds of one sheet of the tissue and rubbed with the tissue, using finger and thumb. The same sheet of tissue can be used for several coverslips. The slip is then sterilized by one or two passages through a flame and placed in a sterile Petri dish to cool.

Operation. The spray is placed in position (see Fig. 2), the plug in its neck removed, and the plate $C$ and glass ring $D$ quickly put in position as previously described. A sterile coverslip is then placed on $D$. When the spray is operated, most of the microdrops which pass through the circular hole in $C$ are collected on the coverslip. If the top of the spray is correctly aligned with the orifice in $C$ and the glass ring $D$, the droplets will be formed over the central area of the coverslip. The number of droplets formed depends on the operator. When the bulb is working efficiently one or two firm squeezes usually suffice. For ease of operation it is an advantage to allow the squeeze bulb to hang over the edge of the bench, which should be of such a height that one can observe comfortably the formation of the droplets by looking down on the top side of the coverslip while operating the squeeze bulb. When sufficient droplets have formed, the coverslip is quickly placed over the well previously filled with liquid paraffin. This slide is now ready for examination under the microscope.

\section{RESULTS}

Pl. 1, fig. 1, gives a visual idea of the droplet size obtained with the technique. As is to be expected, there is a wide variation in size of the microdrops; measurements of droplets formed in this way have ranged from 17 to $170 \mu$. in diameter.

When using a suspension of organisms it is necessary to adjust the density of the suspension by trial and error so that the required number of cells can be obtained in the desired size drop. If the original suspension has been suitably diluted there is usually little difficulty in finding droplets containing the required number of cells, and it is possible to find droplets containing a single cell, e.g. PI. 1, fig. 2. The technique is most useful for organisms which give uniform suspensions, and to ensure this it is sometimes necessary to shake the original suspension with sterile glass beads before placing it in the spray. A total volume of $2-5 \mathrm{ml}$. of suspension is required for the type of spray described.

For organisms which attack liquid paraffin, a small drop of the medium is placed in the bottom of the well. In this case, only the space between the coverslip and edge of the well is sealed with liquid paraffin. In addition, it is an advantage to coat the well with a silicone film to suppress dew-drop formation. Unless the organisms are suspended in distilled water, little diminution in size of the droplets takes place with incubation periods up to 4-5 days (see note). Under these conditions silicone-treated coverslips are much superior to those prepared according to the grease film methods of Reyniers (1933) and Gee \& Hunt (1928). 
At this stage the technique can only be used for direct observations on the growth and development of cells in undisturbed condition. It is possible to fix and stain the cells using the technique described by de Fonbrune (1948) for his oil chambers. However, owing to the presence of the water-repellent film the cells do not settle on the coverglass in their observed position in the drop, but are pulled to one spot as the aqueous drop evaporates through the paraffin. An exception to this is with cells which have hydrophobic properties. For example, it has been observed with the paraffin-decomposing nocardias, e.g. Nocardia salmonicolor, that some cells will migrate to the coverslip at its point of contact with the edge of the drop. Cells in this position will then tend to grow upwards along the air-liquid interface, there being no paraffin in the well. (See also section on growth of $N$. salmonicolor on paraffin droplets.)

In the de Fonbrune method these difficulties do not arise during fixation because the coverglasses used do not need water-repellent films. It is not possible to obtain well-formed droplets on untreated grease-free coverslips with the spray method. The drops formed under these conditions tend to spread out into flat irregular shapes where they hit the coverslip surface. The only way I have been able to fix and stain cells with this method in their original position is to dry in air the untreated coverslips after spraying, and coat them with molten agar media according to the methods of Fleming, Voureka, Kramer \& Hughes (1950). With this method, the initial cells of the resulting microculture are more evenly dispersed than when the suspension is, in the ordinary way, spread over the coverslip. After the necessary incubation, fixed and stained preparations can then be made by any suitable method.

\section{Growth of Nocardia salmonicolor on paraffin droplets}

$N$. salmonicolor is known to grow on liquid paraffin as sole carbon source (Erikson, 1949). A suspension of this organism was made by adding to sterile liquid paraffin containing glass beads, a little of the growth of $N$. salmonicolor from the surface of an agar plate ( $2 \%$ glucose + Czapek salts (nitrate) $+2 \%$ agar). Vigorous shaking by hand was required to obtain a uniform suspension. The paraffin + organism suspension was then introduced into a dry sterile spray. Spraying was carried out in the usual way. The coverslip with its paraffin droplets was then inverted over a sterile well slide, the well being filled beforehand with sterile mineral salt solution (Czapek salts). As the excess mineral salt solution flowed out at the sides of the coverslip it could be taken up with filter paper until the coverslip settled down firmly over the well. Incubation was carried out in moist chambers. When using oils such as liquid paraffin there are very few evaporation troubles; also it is not necessary to apply a water-repellent film to the coverslip beforehand. Pl. 1, fig. 3, shows the growth of the organism after $17-20 \mathrm{hr}$. incubation at $25^{\circ}$ on a paraffin droplet. It was observed that the growth took place at the oil/water interface. A similar experiment was performed in which $1.5 \%$ agar was added to the mineral salt solution; the coverslip was lowered on to the agar medium in the well while the agar was in the molten condition $\left(42^{\circ}\right)$. Pl. 1, fig. 4 , gives the result after 
17-20 $\mathrm{hr}$. incubation at $25^{\circ}$; the organism has grown away from a droplet between the agar and the under surface of the coverslip; connexion with the oil droplet can be clearly seen. The results depicted in Pl. 1, figs. 3 and 4, demonstrate clearly the mycelial type of initial development for this organism (Erikson, 1949). It is interesting to note that it occurs on liquid paraffin. Similar results have been obtained with vegetable oils, e.g. olive oil, almond oil and castor oil.

\section{DISCUSSION}

It must be stressed that the technique presented in this paper is not an alternative for micromanipulation methods. It is obvious that single cell cultures for transplantation cannot be obtained with it, nor is it possible to transfer cells from drop to drop; at present this can only be done by micromanipulation. However, when all that is required is the direct observation of the behaviour and development of a few cells the method should be useful to workers with limited facilities for such purposes at their disposal. In addition, it seems that the technique will be useful for the study of organisms which attack oils.

The author wishes to thank Mrs D. Oxford and Dr G. K. Fraser for their continued interest in this work, and also Miss I. F. Taylor for taking the photographs.

\section{Note}

\section{By V. C. Farmer}

The vapour pressure $p$ of a droplet of radius $r$ is greater than that $\left(p_{0}\right)$ of a plane surface, as given by the equation

$$
\text { 2 } \gamma M / r \rho R T=\log p / p_{0} \bumpeq \Delta p / p_{0},
$$

where $\gamma$ is the surface tension, $\rho$ the density, and $M$ the molecular weight of the liquid, $R$ is the gas constant and $T$ the absolute temperature.

Therefore solvent will be transferred from the droplets on the coverslip to the virtually plane surface of the drop of culture medium in the well of the slide. As the concentration of the solution in the droplet increases, its vapour pressure will fall until equilibrium is reached. Raoult's law gives the change of vapour pressure due to this effect as $\Delta p / p_{0}=-\Delta x$, where $\Delta x$ is the change in mole fraction of the solute.

Thus at equilibrium $\Delta x=2 M / r \rho R T$.

Such changes in temperature $\left(20-40^{\circ}\right)$, density and surface tension (60 to 70 dynes $/ \mathrm{cm}$.) as are likely to occur under experimental conditions will not affect $\Delta x$ by more than $20 \%$. Thus we may calculate that the change in molar fraction will be about $10^{-4}$ for the smallest droplets $\left(r=10 \mu\right.$.) or $10^{-5}$ for the largest $(r=100 \mu$.).

The other important factor is the concentration of the nutrient solution. For instance, the smallest droplets of a $1 \%$ glucose solution (molar fraction $10^{-3}$ ) would undergo a $10 \%$ change in concentration, and the largest only a $1 \%$ change. Any increase in concentration (say by adding inorganic salts) would decrease the effect further. 
Journal of General Microbiology, Vol. 8, No. 1


I. M. Webley-HaNging-Drop merocutaURes. Plate: 1 


\section{REFERENCES}

Erikson, D. (1949). Differentiation of the vegetative and sporogenous phases of the actinomycetes. 4. The partially acid-fast proactinomycetes. J.gen. Microbiol. $3,361$.

Fleming, A., Voureka, A., Kramer, I. R. H. \& Hughes, W. H. (1950). The morphology and motility of Proteus vulgaris and other organisms cultured in the presence of penicillin. J. gen. Microbiol. 4, 257.

De Fonbrune, P. (1948). Technique de Micromanipulation. Paris: Masson et Cie.

GeE, A. H. \& Hunt, G. A. (1928). Single cell techniques. A presentation of the pipette method as a routine laboratory procedure. $J$. Bact. 16, 327.

GiLBERT, JUN., P. T. (1951). Silicone water-repellents for general use in the laboratory. Science, 114, 637.

Reyniers, J. A. (1933). Studies in micrurgical technique. I. The adaption of single cell technique to routine use. $J$. Bact. 26, 251.

\section{EXPLANATION OF PLATE}

\section{Plate 1}

Fig. 1. Sterile hanging microdrops (distilled water under liquid paraffin). $\times 80$.

Fig. 2. Hanging microdrop (distilled water under liquid paraffin) containing a single yeast cell. $\times 680$.

Fig. 3. Nocardia salmonicolor growing on a hanging microdrop of liquid paraffin surrounded with Czapek's salt solution (see text for details).

Fig. 4. As for fig. 3, but with 1.5\% agar added to the mineral salt solution (for details see text).

(Received 27 June 1952) 